\title{
Métodos y tratados de iniciación al estudio del Violín en el siglo XIX
}

\section{Methods and treatises for the learning of Violin in the nineteenth century}

A. Garde Badillo

ana garde badillo@hotmail.com

J. Gustems Carnicer jgustems@ub.edu

Departament de Didàctica de les Ciències Socials, l'Educació Musical, l’Educació Física i l'Educació Visual i Plàstica Universitat de Barcelon

doi: 10.7203/LEEME.39.9903

Recibido: 27-2-2017 Aceptado:9-4-2017.

Contacto y correspondencia: Josep Gustems. Departament de Didàctica de les Ciències Socials, l'Educació Musical, l'Educació Física i l'Educació Visual i Plàstica. Despacho 312. Edifici Levant, $3^{\text {a }}$ planta, Campus Mundet, Universitat de Barcelona. Passeig de la Vall d'Hebron, 171. 08035 Barcelona

\section{Resumen}

En este artículo se presenta una descripción de los principales tratados para la enseñanza del Violín en su fase inicial publicados en el s. XIX a partir del análisis de sus contenidos educativos efectuado mediante una ficha creada y validada para este fin, que contempla aspectos didácticos y metodológicos, habilidades y destrezas motrices y auditivas, refuerzo del lenguaje musical y desarrollo emocional. Se pretende buscar la validez de estos tratados para la actual pedagogía del Violín, destacando los principales elementos presentes en una muestra significativa de catorce tratados del siglo XIX de enseñanza del Violín destinados a alumnos de nivel inicial. Entre los principales contenidos se encuentran la postura, la relajación, la sujeción del Violín y del arco, la graduación de dificultades y la producción de un buen sonido. Dichos avances han llegado a nuestros días y forman parte de los contenidos y procedimientos de enseñanza de este instrumento en la actualidad.

Palabras clave: métodos de Violín; educación musical; métodos musicales; enseñanza del Violín.

\section{Abstract}

This article presents a description of the main treatises for beginning Violin instruction published in the nineteenth century. Based on the analytical file created and validated for this purpose, this description includes didactic and methodological aspects and considers how the treatises deal with motor and hearing skills and abilities, musical language reinforcement and emotional development. The aim of the study is to determine the validity of these treaties for the current pedagogy of the violin, highlighting the main elements present in a significant sample of fourteen treatises for violin teaching, written for students at the initial level, of the nineteenth century. Among the main contents, the volumes include posture, relaxation, the subjection of the violin and the bow, the graduation of difficulties, and the production of a good sound. Those advances have remained sound up to the present time and are part of the contents and teaching procedures of this instrument today.

Keywords: violin methods; musical education; musical methods; violin teaching.

\section{Introducción}

El material didáctico para Violín que se publicó floreció durante todo el siglo XIX, estaba impulsado por las condiciones sociales más favorecedoras, ya que es en esta época en donde se asiste a dos fenómenos de amplia relevancia: 1) el desarrollo de la industria de la publicación y de la edición musical; 2) la fundación de los distintos conservatorios, entidades en donde se va a estructurar la enseñanza de la música. Esto dio paso a un enfoque más sistemático de la educación musical, surgiendo 
la necesidad de un material didáctico que plasmase las necesidades pedagógicas de la época (Triviño, 2015).

El siglo XIX marcó una gran era para el desarrollo técnico del Violín, ya que muchos aspectos que quedaron inconclusos durante el siglo XVIII se desarrollaron y evolucionaron de una manera notable durante este periodo sentando las bases técnicas y clarificando el modo de tocar de un instrumento que en ese momento estaba situado en lo más alto de la jerarquía orquestal. Además, cabe añadir que los autores de los tratados seleccionados en este estudio fueron distinguidos profesores en conservatorios y centros de prestigio musical, así como grandes violinistas que estuvieron en activo dando conciertos y mostrando sus habilidades técnicas. La revolución francesa fomentó la educación pública desarrollándose las grandes escuelas del Violín del siglo XIX (franco-belga y alemana, principalmente). Se fundaron Conservatorios de música que alcanzaron un amplio prestigio, como el de París (1795), donde los profesores creaban sus métodos personales a través de sus programaciones, que posteriormente se convirtieron en fuentes metodológicas de gran calidad y cuyo uso se ha extendido hasta nuestra época.

El objetivo principal de este trabajo es analizar las principales aportaciones a la enseñanza del Violín en su fase inicial durante el siglo XIX, a través de una muestra de métodos y tratados. Para ello, se ha tomado como referencia los principales métodos y autores del siglo XIX que han dedicado sus metodologías a la enseñanza del Violín desde el inicio de los estudios y cuya trascendencia e importancia aún continúa aún vigente. Para el análisis de sus contenidos didácticos, se ha empleado una plantilla creada ad-hoc que incluye una serie de contenidos que abordan tanto aspectos relevantes de la técnica del Violín como otros más generales concernientes a la pedagogía musical y aplicables a la pedagogía del Violín. Dicha ficha ha sido validada por expertos tanto en el campo de la enseñanza de este instrumento como en el de la didáctica musical.

\section{Manuales y tratados de la enseñanza del Violín en la primera mitad del s. XIX.}

La gran mayor parte de los métodos de Violín a principios del siglo XIX estaban destinados a los alumnos principiantes, continuando con el mismo modelo del siglo XVIII (Garde, Gustems y Calderón, 2015), es decir, comenzando con una primera parte introductoria, donde se muestran conceptos básicos de la técnica del Violín (como la colocación de éste y del arco, los diferentes golpes de arco, la técnica de cada mano, etc.) para mostrar a continuación una serie de breves piezas y de ejercicios con una progresividad todavía muy cuestionable, ya que su contenido musical hace imposible que pueda ser abordado por un alumno que se inicia en el estudio del Violín. En sentido parecido, Jorquera (2004) coincide en cómo suelen presentarse los contenidos de los métodos de instrumento.

Uno de los principales métodos de la época fue el Methode de violon, creado y desarrollado en el Conservatorio de París por Baillot, Kreutzer y Rode y revisado posteriormente por Baillot bajo el título de L'art $d u$ violon (Baillot, 1835). Aunque las indicaciones con texto son bastante

THE CONTENT OF THIS ARTICLE IS THE SOLE RESPONSIBILITY OF THE AUTHORS. THE REVISTA ELECTRÓNICA DE LEEME AND UNIVERSITAT DE VALĖNCIA ARE NOT LIABLE FOR ANY LEGAL ACTIONS THAT MAY ARISE INVOLVING THE ARTICLE'S CONTENT. REVISTA ELECTRÓNICA DE LEEME - HTTPS://OJS.UV.ES/INDEX.PHP/LEEME HTTP://MUSICA.REDIRIS.ES ISSN: 1575-9563 EDITORES: UNIVERSIDAD DE VALENCIA Y JESÚS TEJADA GIMÉNEZ. VISIBILIDAD DE ESTA REVISTA: EBSCO, CINDOC (CESIC), CITEFACTOR, COPAC, DIALNET, DICE (CSIC), DOAJ, E-REVISTAS (CSIC), EBSCO PREMIER, ERIH+, GALE CENGAGE LEARNING, IN-RECS, IRESIE, LATINDEX, MIAR, OCLC WORLDCAT, REDIB, RESH, RILM CORE JOURNALS, SUDOC, ULRICHS, ZEITSCHRIFTDATENBANK, ESTA REVISTA ESTÁ PUBLICADA CON EL APOYO INSTITUCIONAL DE UNIVERSITAT DE VALĖNCIA Y REDIRIS-CONSEJO SUPERIOR DE INVESTIGACIONES CIENTIFICAS Y ES DE ACCESO LIBRE. 
desproporcionadas y largas con respecto a su parte más práctica, sin duda se trata de un método que va a representar a la perfección el estado de la pedagogíaa del Violín a partir del siglo XIX. Es el método histórico por excelencia que marcó el principio del desarrollo de la técnica del Violín durante el siglo XIX y su estandarización de la técnica moderna del Violín (Kolneder, 1998),

Resulta muy interesante observar el planteamiento didáctico de estos tres autores, ya en los albores del siglo XIX. Una de las principales premisas del método es tratar de animar e incentivar a los alumnos a través de la técnica y de la mecánica del Violín, para poder dotarse a fondo de una serie de herramientas necesarias para la interpretación musical. Proporciona una serie de estudios muy valiosos que emplearía el principiante como punto de partida, hasta conseguir cierto dominio y poder alcanzar un nivel más profesional.

Otro método destacable de esta primera época fue el método de Bruni (1804). Este es un método muy interesante por varios motivos: uno de ellos es su concepción bastante moderna de sujeción del arco, ya que aboga por una posición más natural, con el codo despegado del cuerpo, resultando una concepción más cercana a nuestra época actual. Otro motivo es su organización bastante estructurada, pues se compone de 107 piezas con acompañamiento de un segundo Violín. Todas estas circunstancias harán de él un libro realmente útil para el desarrollo de la técnica y de la musicalidad.

Es en esta época cuando surgió un original enfoque compositivo en los ejercicios; el nacimiento del concepto de "capricho". Muchos autores recopilaban fragmentos de los conciertos y los reutilizaban variando algunas de sus partes, surgiendo así nuevas piezas musicales con un marcado carácter técnico y un nivel de exigencia superior. Esta idea prosperó durante esta época; por tanto, muchos autores componían estas pequeñas piezas. Este es el caso de los famosos 24 caprichos de Rode o los ya extremadamente virtuosísticos 24 caprichos de Paganini (Kolneder, 1998). Además, hay que hablar de los tipos de textos didácticos que se empleaban para la enseñanza del Violín (así como de la enseñanza de todos los instrumentos): tratados, métodos, estudios y otras formas musicales, como el capricho, etc.

Los modelos de Rode o de Paganini son más cercanos en fundamentación a El clave bien temperado de J.S. Bach. Algunos de estos caprichos son considerados como piezas clave de la formación de un violinista profesional en la actualidad. Un ejemplo de ello se encuentra en los caprichos más famosos y estudiados, gracias a su accesibilidad técnica; tal es el caso de los caprichos 16 y 24 de Paganini, y en los caprichos de Rode: el número 3 (en la segunda posición); número 9 (en la cuarta posición) y el número 10 (en tercera posición). Los caprichos de Rode han perdurado hasta nuestra época como auténticas piezas que desarrollan la técnica del Violín de una forma integral y virtuosística, considerándose la antesala a los caprichos de Paganini.

Kreutzer también compuso pequeñas piezas, aunque con un criterio bastante más lógico y secuenciado que el de Rode o Paganini. En su método, trata de exponer una serie de estudios progresivos en los que pudo plasmar los principios básicos de la técnica del Violín. Su método, con su revisión y ampliación de 40 a 42 estudios, resulta desorganizado y complejo, aunque sigue siendo el método más trabajado y considerado más importante dentro del estudio del Violín en la actualidad y en general en toda la historia de la pedagogía violinística. 
Viena fue otro de los centros de publicación y de edición de métodos gracias a su amplia tradición en la música orquestal y camerística, lo que hizo que se prodigara la publicación de una gran cantidad de material destinado a la didáctica del Violín. Es en este contexto donde surgen obras como: el método de Kreutzer, Dont, o Hellmesberger (Stowell, 1992).

\section{Manuales y tratados de la enseñanza del Violín en la segunda mitad del s. XIX.}

A mediados del siglo XIX, aparecen manuales mucho más asequibles y progresivos destinados a principiantes, donde se ayuda al estudiante a resolver sus problemas técnicos. Un ejemplo de este intento de facilitar el aprendizaje a los alumnos que se inician en el estudio del Violín se puede encontrar en Dancla (1855), autor que realizó una metodología plasmada en varios libros didácticos. En ellos trata y justifica su insatisfacción con los materiales didácticos existentes a principios del siglo XIX. Realiza además en el prefacio de su método una crítica a su maestro Baillot, del cual se encargó de reeditar y revisar el Methode de violon (Kreutzer, Rode y Baillot, 1802-03). Dancla expresa que es necesario un método adicional a este para poder afrontar el estudio de la reedición proporcionada por Baillot (Dancla, $1855)$.

Hay métodos, como el de Habeneck (1842), que no han conservado un lugar tan privilegiado en la actualidad debido quizás a su extensa parte teórica al comienzo del método. Otros han formado parte en la actualidad de todos los planes de estudio universales dentro de los Conservatorios y centros musicales actuales. Estos son, sin duda, los métodos de Kayser (1848), Dancla (1855) y Mazas (1843).

Dentro de esta misma línea pedagógica y destinada a los principiantes está el método de Alard (1846) y sobre todo el de De Bériot (1858), un representante de la escuela franco-belga, que trataría de enseñar una evolución natural en la técnica del Violín, a diferencia de la escuela italiana, influida por Paganini. Se trata de un método que ofrece ejercicios propedéuticos donde se prepara al principiante desde un primer momento para tratar de alcanzar un nivel técnico adecuado a las circunstancias del momento y de las obras de la época. De Bériot toma elementos de la técnica vocal y los aplica a la técnica del Violín. En su método, aparecen términos como "port de voix" que traducido, sería el portamento en el canto.

Otros grandes métodos de la época fueron los de Dont (1849) y el de Hohmann (1840), primer tratadista cuya obra está destinada plenamente al grado inicial o a las enseñanzas elementales de música. Su método de Violín, perfectamente organizado y estructurado, está ordenado en cinco volúmenes y ha sido traducido a muchos idiomas, convirtiéndose en uno de los libros de referencia del Violín para principiantes de esta época.

Muchos de los métodos para principiantes eran de origen alemán. Esto es debido a que a mediados del siglo XIX la enseñanza del Violín formaba parte de los planes de estudio de la asignatura de música en los centros de Educación Primaria, cuyo plan de estudios pretendía elevar el nivel y el gusto musical a través del aprendizaje del Violín. Posteriormente, se desarrolló con otros instrumentos, 
lo que condujo a la publicación de una gran cantidad de obras pedagógicas destinadas a los centros de Educación Primaria. Un ejemplo de esta metodología se encuentra en el método de Tottmann (1874).

La aportación de los tratadistas alemanes está justificada gracias a su intensa tradición musical, así como su amplia actividad cultural. Es por este motivo por el que hay que mencionar el tratado de Spohr (1852), gran virtuoso y referente en Alemania. Con su tratado es posible desarrollar un amplio dominio del instrumento.

Tabla 1. Resumen de las principales aportaciones de los métodos seleccionados

\begin{tabular}{|c|c|c|}
\hline $\begin{array}{l}\text { Método histórico que } \\
\text { marcó la técnica del } \\
\text { Violín en el s. XIX. }\end{array}$ & $\begin{array}{l}\text { P. Baillot, P. Rode y R. Kreutzer: Methode } \\
d u \text { violon, } 1802-03 \text {, París }\end{array}$ & $\begin{array}{l}\text { Primer Tratado que plantea la técnica del } \\
\text { arco a partir de las novedades introducidas } \\
\text { por Tourte, el constructor de arcos. }\end{array}$ \\
\hline \multirow{2}{*}{$\begin{array}{l}\text { Métodos de los grandes } \\
\text { maestros del Violín de } \\
\text { principios s. XIX }\end{array}$} & $\begin{array}{l}\text { R. Kreutzer: } 42 \text { etudes ou caprices pour le } \\
\text { violon, 1807, París }\end{array}$ & $\begin{array}{l}\text { Estudios de gran valor didáctico. } \\
\text { Profundizan en la técnica integral del } \\
\text { instrumento. }\end{array}$ \\
\hline & P. Baillot: L'art du violon, 1835, París & $\begin{array}{l}\text { Desarrollo de la técnica a través del } \\
\text { repertorio seleccionado y de la } \\
\text { musicalidad. }\end{array}$ \\
\hline \multirow{3}{*}{$\begin{array}{l}\text { Métodos de mayor } \\
\text { perdurabilidad } \\
\text { relevancia dentro del } \\
\text { panorama de la } \\
\text { pedagogía del Violín en } \\
\text { la actualidad. }\end{array}$} & $\begin{array}{l}\text { H. E. Kayser: } 36 \text { Etudes, Op. } 20 . \\
\text { Elementary and progressive studies for the } \\
\text { violin, } 1848, \text { Nueva York. }\end{array}$ & $\begin{array}{l}\text { Estudios preparatorios para abordar otras } \\
\text { obras didácticas de mayor envergadura. }\end{array}$ \\
\hline & $\begin{array}{l}\text { C. Dancla: Methode elementaire et } \\
\text { progressif du violon, Op. } 52,1855, \text { Paris. }\end{array}$ & $\begin{array}{l}\text { Ejercicios secuenciados y progresivos con } \\
\text { validez en la actualidad. }\end{array}$ \\
\hline & $\begin{array}{l}\text { J. F. Mazas: } 75 \text { études mélodiques et } \\
\text { progressives, Op. } 36, \text { vol. } 1,1843 \text {, } \\
\text { Leipzig. }\end{array}$ & Estudios que desarrollan la musicalidad. \\
\hline \multirow{4}{*}{$\begin{array}{l}\text { Métodos destinados } \\
\text { exclusivamente a la } \\
\text { pedagogía del Violín en } \\
\text { la etapa inicial. }\end{array}$} & D. Alard: Ecole du violon, 1846, París. & $\begin{array}{l}\text { Da mucha importancia al estudio diario de } \\
\text { las escalas. }\end{array}$ \\
\hline & $\begin{array}{l}\text { J. Dont: } 24 \text { exercices pour le violon } \\
\text { preparatorias aux Etudes } R \text {. Kreutzer y } \\
\text { P. Rode, } 1849 \text {, París. }\end{array}$ & $\begin{array}{l}\text { Recopilatorio de ejercicios preparatorios a } \\
\text { los métodos de Kreutzer y Rode. }\end{array}$ \\
\hline & $\begin{array}{l}\text { C. A. De Bériot: Methode de violon, Op. } \\
\text { 102, vol. 1, 1858, París. }\end{array}$ & $\begin{array}{l}\text { Método muy progresivo y estructurado. } \\
\text { Nuevo enfoque de las escalas a través de } \\
\text { pequeñas melodías. }\end{array}$ \\
\hline & $\begin{array}{l}\text { F. Wohlfart: Sixty Studies for the violin, } \\
\text { Op. } 45 \text {, Book I, 1874, Nueva York. }\end{array}$ & $\begin{array}{l}\text { Estudios para el desarrollo de la técnica } \\
\text { del Violín, muy progresivos y } \\
\text { secuenciados. }\end{array}$ \\
\hline $\begin{array}{l}\text { Métodos diseñados para } \\
\text { el desarrollo de una } \\
\text { habilidad o destreza } \\
\text { técnica }\end{array}$ & $\begin{array}{l}\text { H. Schradiek: Die as hule der } \\
\text { Violintechnik, 1875, Hamburgo. }\end{array}$ & $\begin{array}{l}\text { Desarrollo integral de la articulación de la } \\
\text { mano izquierda. }\end{array}$ \\
\hline \multirow{2}{*}{$\begin{array}{l}\text { Métodos de los } \\
\text { tratadistas alemanes }\end{array}$} & L. Spohr: Violinschule, 1832, Viena. & $\begin{array}{l}\text { Primer método que introduce la barbada } \\
\text { del Violín. }\end{array}$ \\
\hline & F. David: Violinschule, 1863, Nueva York. & $\begin{array}{l}\text { Empleo de diferentes movimientos de } \\
\text { danzas. }\end{array}$ \\
\hline $\begin{array}{l}\text { Método sistemático e } \\
\text { integral de la técnica del } \\
\text { Violín }\end{array}$ & $\begin{array}{l}\text { O. Sevcik: Die as hule der Violintechnik, } \\
\text { 1875, Hamburgo. }\end{array}$ & $\begin{array}{l}\text { Libro de técnica con indicaciones del } \\
\text { diapasón para saber qué posición tendrán } \\
\text { los dedos. }\end{array}$ \\
\hline
\end{tabular}


En la misma línea, destaca David (1863), su discípulo. Su tratado es una síntesis de la pedagogía y de su didáctica llevada a cabo en el conservatorio de Leipzig, otro de los centros de mayor relevancia con respecto a la pedagogía del Violín durante esta época (Brown, 2007). Otro método bastante representativo de la época fue el de Schradieck (1875). Este autor se centró principalmente en el desarrollo de la articulación de la mano izquierda, siendo el libro de referencia en este sentido que aún se sigue utilizando en la actualidad.

Dentro de la tradición violinística centro-europea, destaca Sevcik (1881). Este autor revolucionó el estudio de la técnica del Violín, elaborando una sistematización metodológica e integral de la técnica básica del Violín, con un carácter bastante científico y basado en el sistema diatónico de afinación. Además, elaboró un análisis científico exhaustivo de los elementos de la ejecución violinística, desarrollando una metodología compuesta por una serie de libros didácticos cuyos ejercicios están, destinados al control absoluto de la técnica, tanto para alumnos de enseñanzas elementales, intermedias como superiores. Es sin duda un autor que culminó el siglo XIX y a la vez enlazó con el siglo XX.

\section{Método}

\subsection{Muestra}

Los tipos de métodos del siglo XIX seleccionados y los criterios de selección han sido:

- El método histórico por excelencia que marcó el principio del desarrollo de la técnica del Violín durante el siglo XIX, surgido en el Conservatorio de París.

- Los métodos de los grandes maestros del Violín de principios del siglo XIX.

- Métodos de mayor relevancia dentro del panorama de la pedagogía del Violín y que mayor perdurabilidad han tenido a lo largo de la Historia, formando parte en la actualidad de todos los planes de estudio de los Conservatorios y centros musicales.

- Los tratados destinados exclusivamente a la pedagogía del Violín en su fase inicial.

- Los métodos diseñados para el desarrollo de una habilidad o destreza violinística.

- La aportación de los tratadistas alemanes.

- Una de las metodologías más sistemáticas e integrales de la técnica del Violín.

Por lo tanto, siguiendo estos apartados, los métodos elegidos para su análisis han sido: los de Kreutzer, Rode y Baillot (1802-3); el de Baillot (1835); los de Kayser (1848), Dancla (1855) y Mazas (1843); Alard (1846) y De Bériot (1858); los de Dont (1849), Wolfhart (1874) y Hohmann (1840); el de Schradiek (1875); el de Spohr (1852) y su discípulo David (1863); y el método de Sevcik (1881). 


\subsection{Instrumento}

Este trabajo parte del método descriptivo a través del análisis documental. Se ha aplicado una ficha construida ad hoc que recoge los contenidos conceptuales, procedimentales y actitudinales presentes en cada uno de los tratados y métodos seleccionados. Esta ficha se ha inspirado en el modelo paramétrico de Frega (1997) y en el de Domínguez (2002) así como de otras fichas similares1 y ha sido triangulada mediante las aportaciones de profesionales del Violín, profesores de Violín y pedagogos musicales2.

El orden en que está dispuesto dicha ficha (Garde y Gustems, 2014) parte de cinco ejes fundamentales, dentro de los cuales están incluidas las siguientes competencias:

- Eje didáctico-Metodológico: de lo simple a lo complejo.

- Habilidades y destrezas motrices: relajación, colocación del Violín, sujeción del arco, trabajo con el arco, posición de la mano derecha, colocación de la mano izquierda, etc.

- Destrezas y habilidades auditivas: buen sonido, afinación.

- Destrezas relacionadas con el lenguaje musical.

- Incluye aspectos cognitivos y expresivos: memoria, interpretación.

\section{Resultados}

Siguiendo la estructura de la ficha de análisis, se presentan a continuación los principales resultados obtenidos en el análisis de contenido de la muestra de métodos del s. XIX.

\subsection{Eje didáctico -metodológico}

De lo simple a lo complejo

Tras el análisis de los métodos propuestos, se ha podido observar que la mayor parte de los métodos analizados pretenden acceder a sus contenidos a través de una progresión donde impere la pauta "de lo simple a lo complejo". Sin embargo, muchas veces dicha progresividad realiza saltos importantes, dejando bastantes lagunas en algunos temas y exponiendo otros de una forma bastante sofisticada y compleja.

De los métodos analizados, los más adecuados de acuerdo al parámetro didáctico-metodológico serían el método de Alard (1846) y el de De Bériot (1858). Ambos métodos trazan una línea progresiva, cuyos ejercicios van introduciendo poco a poco las dificultades y no hacen ningún salto en las dificultades en lo que respecta a la técnica del Violín. Ambos tratados pueden ser empleados en la

\footnotetext{
${ }^{1}$ La ficha completa puede consultarse en http://hdl.handle.net/2445/60685

${ }^{2}$ El proceso de construcción y validación puede consultarse en http://www.tdx.cat/handle/10803/397644 pp. $173-182$.
} 
actualidad, y sin duda con un gran éxito entre los principiantes y los alumnos de las enseñanzas elementales.

\subsection{Habilidades y destrezas motrices}

\section{Relajación y colocación general}

La gran mayor parte de los métodos, exceptuando algunos de ellos como los de Kreutzer (1798 y la versión de 1809 con su ampliación a 42 estudios) y Schradiek (1875), destinan un apartado a explicar algunos aspectos concernientes a conseguir determinadas habilidades físicas.

El método de Baillot (1835) da bastantes pautas acerca de la adecuada relajación del cuerpo a la hora de tocar el Violín, continuando con la misma línea que el Méthode de violon (Baillot, Kreutzer y Rode, 1802-03). Sin embargo, es aún más preciso, ya que el autor propone 7 artículos en que describe las normas básicas de sujetar el Violín y el arco, así como también del estado de equilibrio del cuerpo.

Los tres autores citados (Kreutzer, Rode y Baillot) abogan por el hecho de que una noble y relajada postura favorece el desarrollo de las habilidades motrices, incrementando el encanto de la interpretación y reforzándola para que no suponga un esfuerzo extraordinario la acción de tocar el Violín en sí misma.

Además, Baillot (1835) recomienda utilizar un espejo para observar si se está teniendo una adecuada posición, es decir, si el Violín está colocado correctamente, si la articulación de los dedos es uniforme, si el arco está teniendo una conducción adecuada, etc. Este autor sorprende proponiendo y explicando la manera correcta de tocar sentado:

"El peso del cuerpo se sostendrá sobre la pierna izquierda y los pies deberán de situarse a la misma altura. La flexión de la pierna derecha ligeramente hacia dentro con el fin de evitar el contacto del codo del brazo derecho con la rodilla del mismo lado" (Baillot, 1835, p. 11).

Baillot aconseja también el uso de un taburete como soporte de la pierna izquierda, de tal manera que el cuerpo se mantenga recto. Este planteamiento es contrario al método de Hohmann (1840), quien plantea que es bastante mejor tocar de pie que sentado. Alard (1846) añade que el cuerpo debe tener aplomo y debe conservar una posición equilibrada.

Otro autor que da importancia al hecho de tocar de pie es Dancla (1855), quien además insiste en la actitud del violinista frente al atril, tratando de que la voluta del Violín apunte hacia la esquina superior derecha del atril. 
LISTA ELECTRÓNICA EUROPEA DE MÚSICA EN LA EDUCACIÓN

\section{Colocación del Violín}

Todos los métodos analizados coinciden en que el Violín se coloca encima de la clavícula y se sujeta con el mentón o barbilla, que se apoya en el lado izquierdo del cordal. Sin embargo, a medida que los métodos se acercan hacia finales del siglo XIX se busca que la presión de la barbilla sea más estable y deje mayor libertad a la mano izquierda para poder desarrollar una técnica más refinada y de mayor rapidez, comenzando por Baillot en 1835. Este autor describe de una manera bastante secuenciada de cómo se sujeta el Violín (ver figura 1):

"El violín se debe sujetar sobre la clavícula izquierda, inclinándolo hacia la derecha aproximadamente a 45 grados, sujetado firmemente por la barbilla y lo más cercano al cordal. El mentón se apoya en el violín pero no en el cordal. La barbilla no debe sobresalir y tiene que sujetar el violín sin demasiada presión" (Baillot, 1835, p. 11).

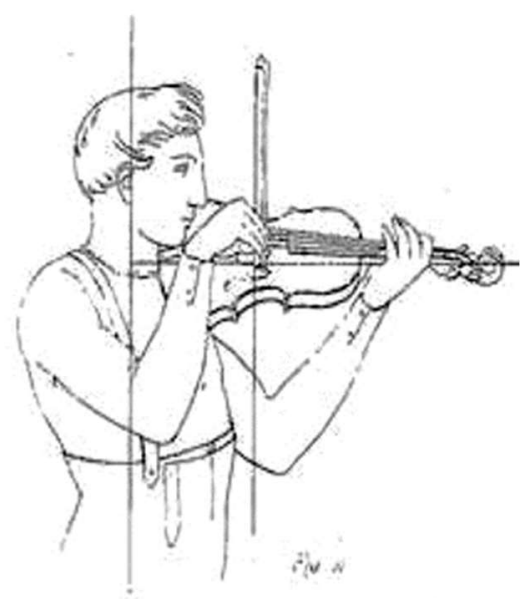

Figura 1. Colocación del Violín (Baillot, 1835).

En el año 1820, se produce uno de los hechos que mayor trascendencia han tenido para la acción de sujetar el Violín. Este fue la creación de la barbada por Spohr, pequeña pieza de madera situada a la izquierda del cordal y que sirve de soporte a la barbilla. Originalmente, se diseñó para ser colocada sobre el cordal en el centro del Violín. Era una guía para colocar la barbilla en su correcta posición, el lado izquierdo. El mismo autor expresa "Si el alumno deseara apoyar y descansar su barbilla en la barbada, entonces, podrá ser colocada a la izquierda del cordal" (Spohr, 1852, p. 24).

Con la gradual aceptación de la barbada en el lado izquierdo del cordal, la posición del codo y del brazo izquierdo fue necesariamente modificada, requiriendo una posición relajada y alejada del cuerpo para poder mantener el instrumento horizontal. El codo izquierdo se colocaría verticalmente por debajo del Violín, justamente coincidiendo con la mitad del mismo (ver figura 2). Esta técnica fue 
propuesta en todos los métodos analizados de esta época: "El codo del brazo izquierdo deberá de ser dibujado hacia el interior hasta que se encuentre con el centro del violín" (Spohr, 1852, p. 13).

En cuanto a la colocación de los pies, existe también una ligera controversia, ya que autores como Alard (1846), Baillot (1835), David (1863) o De Bériot (1858) sitúan el pie derecho ligeramente adelantado al pie izquierdo. Otros como Hohmann (1840) establecen que es mejor adelantar el pie izquierdo de tal manera que este se mantenga en la misma línea, con el codo izquierdo estando los talones alineados y a una corta distancia.
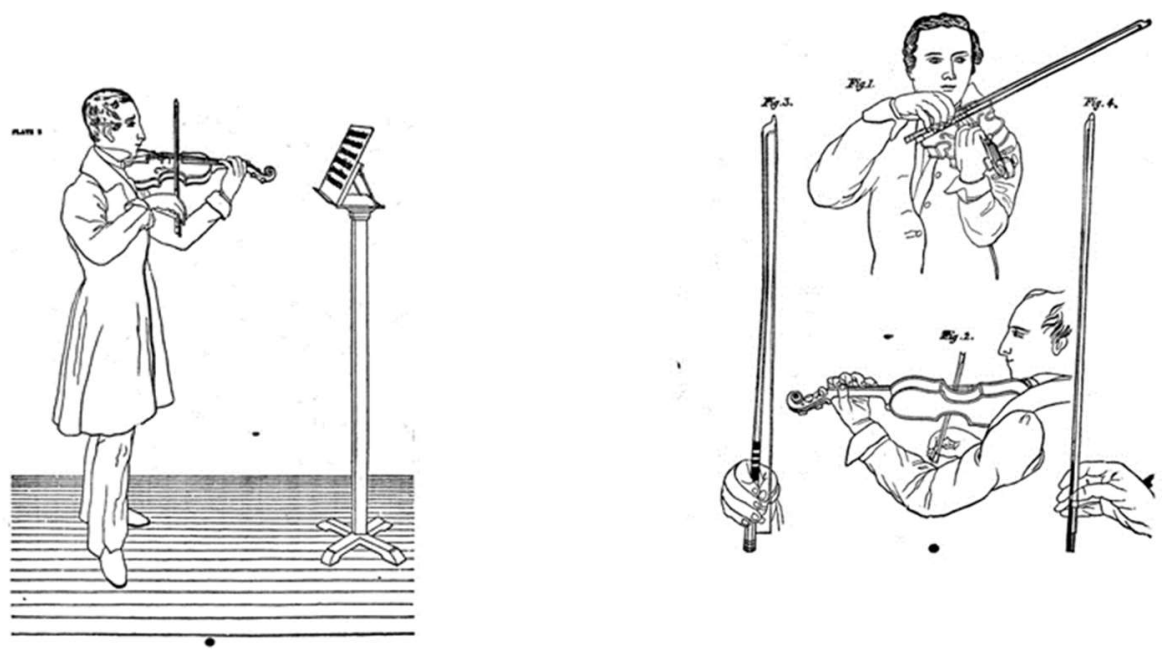

Figura 2. Correcta colocación postural (Spohr, 1852).

Sujeción del arco

Gracias a la aparición del famoso arco de Tourte, se estandarizó la técnica del arco, sobre todo en cuanto a saber la situación de cada dedo, su lugar y la función que desempeñan. Francoise Tourte (1747-1835) era un prestigioso fabricante francés de arcos, quien definió su forma definitiva. Anteriormente la curvatura de la madera era cóncava y con mayor tensión en las cerdas (ver figura 3). Uno de los maestros que promovió el uso del arco continuo fue Giovanni Battista Viotti (1755-1779) dando lugar a un movimiento continuo del brazo derecho. Viotti trabajó junto a Tourte creando un arco más largo que el anterior existente y un mayor peso en los extremos.

En el talón, incorporó una pieza metálica o tornillo, que servía para dar mayor tensión a las cerdas o crines; además facilitaba que las cerdas estuvieran planas y uniformes en contacto con las cuerdas, produciendo mayor volumen y estabilidad. Estas modificaciones provocaron un auténtico cambio en la manera de tocar, ya que permitían tocar arcadas más largas, con legato, con claridad y con rapidez (Prieto, 1998). 


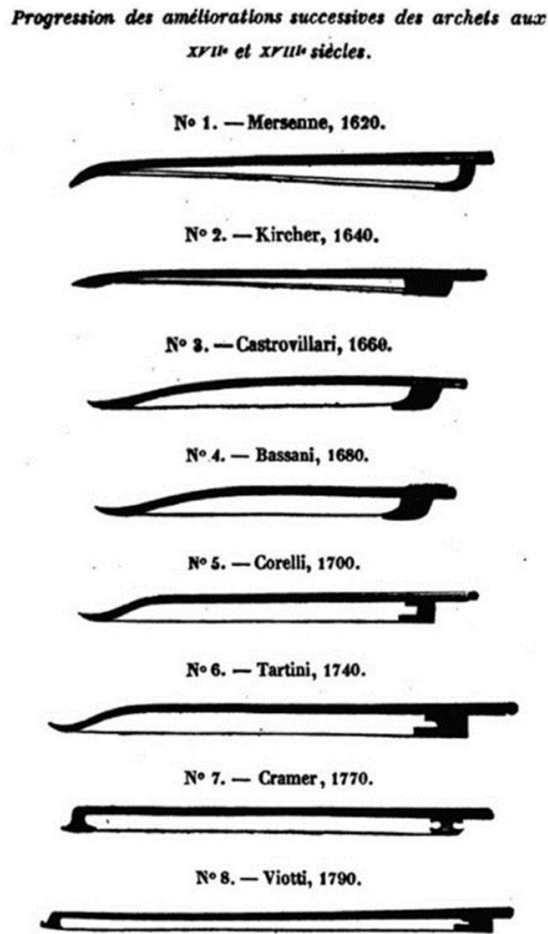

Figura 3. Evolución de la curvatura del arco (Museo de Bellas Artes de Viena)

Posición de la mano derecha y el rol de los dedos

Todos los métodos analizados plantean este aspecto y coinciden en sujetar el arco con todos los dedos, que el pulgar esté situado en la nuez del arco y en oposición con el dedo corazón formando el dibujo de un círculo. El índice se recostará encima de la vara a la altura de la segunda falange.

\section{Conducción del arco}

Todos los métodos analizados que describen este concepto coinciden en que la vara debe estar ligeramente inclinada hacia el diapasón y el arco tiene que pasar paralelo al puente. 


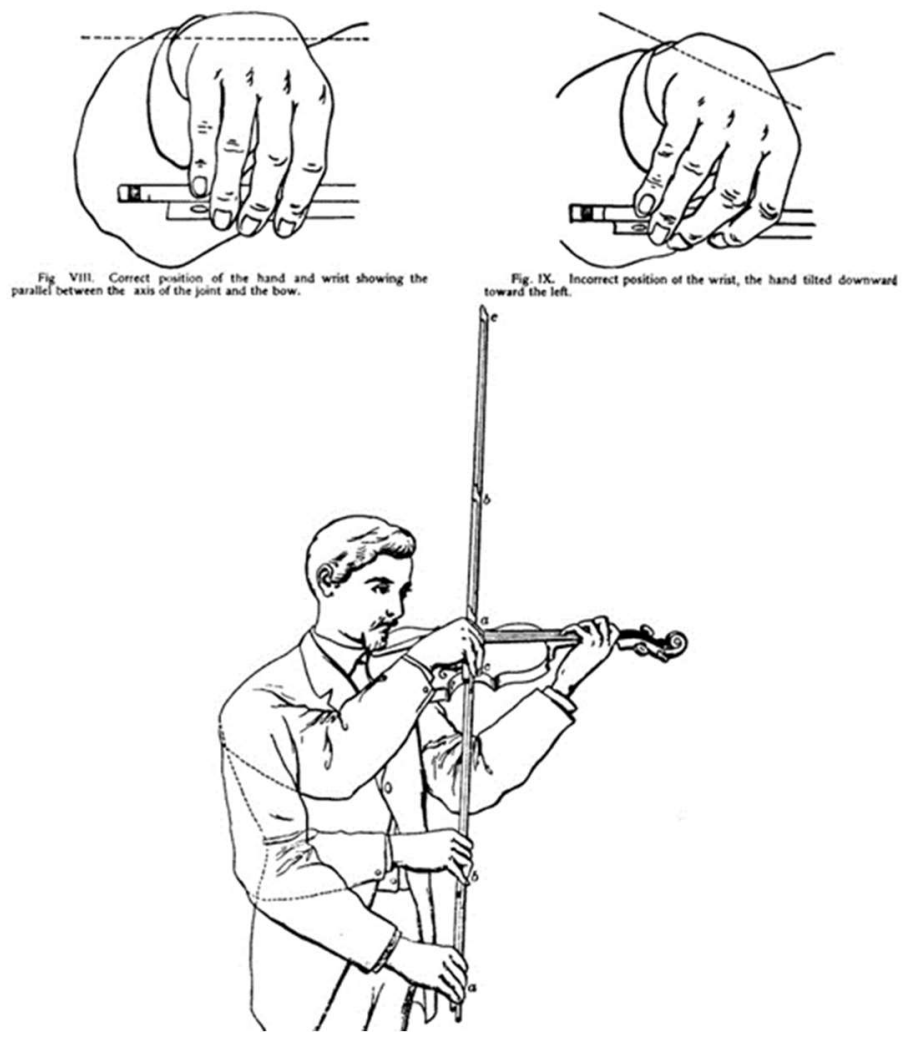

Fig. 4. Colocación de la mano derecha y conducción del arco (De Bériot, 1858)

\section{Distribución del arco}

Según Kreutzer, Rode y Baillot (1802-3), el arco se debe conducir de un extremo al otro. Aconsejan que el meñique deba soportar el peso del arco sobre el talón, colocándolo bastante flexionado y sin rigidez. El codo sigue el movimiento de la mano. Baillot (1835) y Alard (1846) dividen el arco en tres secciones: talón, centro y punta utilizando el dibujo de un arco y señalando en cada ejercicio la zona donde se debe tocar.

\section{Cambios de cuerda}

La mayor parte de los tratados señalados coinciden en que el paso de una cuerda a otra no debe notarse, tratando que el brazo no haga un movimiento brusco. Para ello plantean la importancia de mantener la muñeca ligeramente flexionada y el codo ligeramente elevado por encima de la muñeca, a excepción del método de De Bériot (1858), que plantea que el codo y la muñeca estarán a la misma altura y el hombro deberá estar relajado. 
LISTA ELECTRÓNICA EUROPEA

DE MÚSICA EN LA EDUCACIÓN

\section{Posición de la mano izquierda}

Todos los métodos analizados coinciden en apoyar el Violín entre la primera falange del pulgar y la tercera del dedo índice, quedando el mango del Violín lejos de la parte en donde se unen ambos dedos (pulgar e índice). Hohmann (1840) añade que la palma de la mano estará alejada del cuerpo del Violín, la muñeca curvada hacia el exterior y el codo hacia dentro y debajo del cuerpo del Violín. David (1863) añade que la muñeca deberá formar una línea con el codo y con los dedos de la mano izquierda.

Todos los métodos continúan con la línea del famoso tratado de Geminiani (1751), que ya planteó una forma estándar de colocación de la mano izquierda y que continúa utilizándose durante todo el siglo XIX. Esta nueva manera fue denominada "el agarre de Geminiani".

\section{Manner of Holding the Left Hand.}

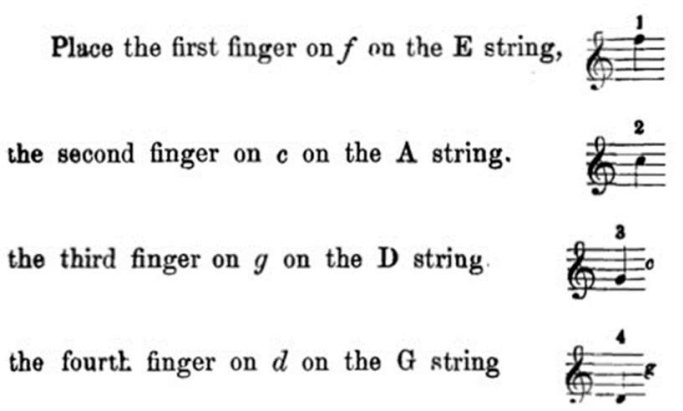

Figura 5. Colocación de la mano izquierda a la manera de Geminiani (David, 1863)

\section{Articulación de los dedos}

Todos los métodos del s. XIX analizados coinciden en que los dedos deben moverse con flexibilidad y uniformidad, alzándolos suficiente para que puedan apretar la cuerda con la suficiente presión y fuerza. De Bériot (1858) puntualiza que los dedos de la mano izquierda deben estar preparados para apoyarse en cualquier momento sobre las cuerdas, tratando de que no aplasten las cuerdas adyacentes en una posición curvada y lo más verticalmente posible hacia la cuerda.

Hohmann (1840) plantea en su método que los dedos deben apoyarse con la punta de la yema, dando especial importancia al trabajo del cuarto dedo. Añade que se tiene que evitar la articulación de los dedos, lo más similar a un pianista. Schradieck (1875) es el autor de un método destinado a trabajar únicamente el concepto de la articulación. En su única pauta descriptiva al comienzo de su método, recomienda: "Trabajar los ejercicios con la mano bastante relajada, los dedos deben ser vigorosos y con elasticidad. Trabajar lentamente para posteriormente ir ampliando la velocidad" (Schradieck, 1875, p. 1). 
De Bériot (1858) y Alard (1846) dan bastante importancia al estudio de las escalas para desarrollar una correcta articulación. Según De Bériot, los dedos deben estar preparados para caer en la siguiente cuerda lo más rápido posible y con la mayor anticipación. Los dedos se deben mantener en la misma posición, el mayor tiempo posible, evitando una actividad innecesaria que conlleve pérdidas de energía. Esta técnica se aplica en pasajes que requieren de cierta rapidez de movimientos, evitándose en otros con notas largas, ya que se podría agarrotar la mano. Los dedos se mantienen a distancia de una pulgada aproximadamente de la cuerda.

\section{Cambios de posición}

En cuanto a los cambios de posición se recomienda deslizar la mano por el mango del Violín. Dan importancia al trabajo de este aspecto a través de ejercicios prácticos, pero ninguno de los métodos analizados explica de forma detallada y descriptiva cómo deben realizarse de forma adecuada los cambios de posición.

\section{Extensiones}

El único método analizado que aborda este tema de forma muy descriptiva es el método de Baillot (1835). Este da la pauta de extender el cuarto dedo, sin necesidad de mover toda la muñeca para llevar a cabo la extensión.

\section{Dobles cuerdas}

El método que traza una línea bastante progresiva y organizada respecto a las dobles cuerdas es el método de Sevcik (1881), que utiliza las cuerdas al aire para comenzar a practicarlas y posteriormente va añadiendo cada dedo. De Bériot (1858) también argumenta que para empezar el trabajo del estudio de las dobles cuerdas, se debe comenzar por realizarlas en cuerdas al aire. Baillot (1835) da la única, aunque incompleta, pauta de colocar el arco cerca del diapasón para realizar dobles cuerdas, y para los acordes, -acercar el arco a la tercera cuerda-.

\section{Trabajo con el trino}

De todos los métodos analizados, el más exhaustivo en este sentido ha sido el de Kreutzer (1799/1809); sin embargo, sus ejercicios prácticos son demasiado difíciles para empezar a trabajar con principiantes. Alard (1846), al contrario que Kreutzer, da una serie de pautas para principiantes sobre cómo realizar y trabajar el trino. Para ello, recomienda dejar los dedos con extrema flexibilidad y practicar lentamente para conseguir un movimiento lo más igualado posible. 
LISTA ELECTRÓNICA EUROPEA

DE MÚSICA EN LA EDUCACIÓN

\subsection{Destrezas y habilidades auditivas}

\section{Producción de un buen sonido}

Baillot (1835) plantea la importancia de dar un buen punto de contacto al arco, de manera que no se ejerza una excesiva presión sobre las cuerdas que haga que el sonido se rompa. De Bériot (1858), además, recomienda sostener el sonido al máximo, conectando el arco arriba con el arco abajo y tratando de no dar un impulso innecesario en el cambio de arco.

Hohmann (1840), por su parte, establece que para obtener un buen sonido se debe tener en cuenta la inclinación del arco, pasar el arco paralelo al puente y que el codo esté a la altura de la muñeca sin subir el hombro, que se debe mantener relajado. Por último, en cuanto a la velocidad del arco, Dancla (1855) da bastante importancia a comparar el sonido de las notas pisadas con los cuatro dedos.

Respecto a la amplificación del sonido, este tema está bastante desarrollado en los tratados del siglo XIX, ya que se trata de jugar con la amplitud y disminución del sonido para conseguir diferentes efectos y recursos expresivos, de tal manera que si el arco se acerca al puente se obtendrá un sonido más forte y si se aleja será más piano o suave.

\section{Concepto de la afinación}

Hohmann (1840) plantea afinar primero la cuerda de LA, y después afinar y comparar el resto de cuerdas. Spohr (1852) propone que el Violín del principiante debe estar perfectamente afinado por el profesor para que éste adquiera la concepción de una buena entonación. De Bériot (1858) utiliza el estudio de las dobles cuerdas para poder escuchar de una manera más precisa la afinación por quintas.

\subsection{Refuerzo del lenguaje musical/ lectura y ritmo}

Pocos son los métodos que den unas nociones de estos aspectos durante el siglo XIX; no obstante, algunos métodos como el de Spohr (1852) realizan una introducción a ciertos conceptos básicos del lenguaje musical.

\subsection{Desarrollo emocional y afectivo}

Ninguno de los tratados del siglo XIX propuestos para el análisis desarrolla conceptos como la motivación del profesor, la implicación personal o inclusive la implicación familiar, etc. Es decir, probablemente se trataría de aspectos poco relevantes en aquella época, dando más importancia a otros que estaban más en relación con el arte de la interpretación en sí misma. El único que habla directamente de la motivación al alumno, que viene dada por el profesor, es el método de Wolfhart (1874). Este autor 
plantea la necesidad de motivar a los alumnos a realizar un buen trabajo con el instrumento, aconsejando realizar ejercicios musicales motivadores.

\section{Conclusiones}

En este trabajo, se han seleccionado los métodos del s. XIX que más relevancia han tenido para la historia de la pedagogía del Violín en su fase inicial, se han buscado métodos que han perdurado hasta nuestra época, encontrándonos con diferentes problemas en su análisis, como por ejemplo el hecho de plantear metodologías muy complejas y de un nivel bastante superior y virtuoso aunque de gran valor e importancia para la historia de la pedagogía del Violín, excepto el método de Rode y el de Mazas, (totalmente virtuosísticos y que no siguen la línea de esta investigación).

Otro problema al analizar estos tratados reside en que muchos de ellos no cuentan con descripciones o indicaciones, siendo a menudo una mera selección de ejercicios progresivos donde el autor no expone ningún criterio teórico ni explica la intención de cada pieza o estudio mostrado. Este es el caso de Dont (1849), Kayser (1848), o Wolfhart (1874), aunque la mayoría suelen evitar explicaciones. Sin embargo, sus contenidos prácticos pueden resultar valiosos para el estudio y el análisis de los tratados del siglo XIX, como el caso de Schradieck (1875) y de Sevcik (1881).

El siglo XIX fue una época de clarificación para la técnica del Violín, igual que para muchos otros instrumentos, ya que muchos de los aspectos técnicos planteados durante el siglo XVIII fueron resueltos a partir de esta época formando conceptos importantes que perdurarán en nuestra época actual y sentarán las bases de la técnica actual del Violín. Esta estandarización técnica, llevó a muchos autores a ser poco teóricos e instructivos en sus manuales, dando por hecho que el profesor debería conocer y entender los objetivos que cada autor pretendía lograr con un determinado ejercicio, pieza musical o ejemplo práctico (Garde, Gustems y Calderón, 2015). Es por este motivo, por el que existe una gran diferencia entre los tratadistas y teóricos del siglo XVIII y los autores del siglo XIX. Estos últimos eran violinistas, virtuosos y profesores de Violín, que pretendían plasmar su forma o manera de enseñar sus conocimientos técnicos y compositivos y sus logros en cuanto a destrezas y mecanismos técnicos.

Tras el análisis de los tratados se puede decir que el siglo XIX marcó una gran era para el desarrollo técnico del Violín, ya que muchos aspectos que quedaron inconclusos durante el siglo XVIII se desarrollaron y evolucionaron de una manera notable. En sus métodos muestran de una manera muy exhaustiva aspectos que están relacionados con la colocación del instrumento y del arco. Sin embargo, dejan otros aspectos bastante poco definidos, como es el caso del control corporal. Todos explican que se debe tener una adecuada actitud para poder enfrentarse con el instrumento, sin embargo, ninguno menciona aspectos como la relajación para tocar el Violín.

Otra observación tras el análisis está directamente relacionada con el hecho de que se trata de manuales donde lo que se pretende es orientar al alumno a que domine todos los recursos técnicos. Comienzan con una serie de ejercicios básicos, hasta llegar progresivamente a desarrollar todas las posibilidades sonoras del instrumento a través de las piezas y de los ejemplos prácticos que exponen.

THE CONTENT OF THIS ARTICLE IS THE SOLE RESPONSIBILITY OF THE AUTHORS. THE REVISTA ELECTRÓNICA DE LEEME AND UNIVERSITAT DE VALĖNCIA ARE NOT LIABLE FOR ANY LEGAL ACTIONS THAT MAY ARISE INVOLVING THE ARTICLE'S CONTENT. REVISTA ELECTRONICA DE LEEME - HTTPS://OJS.UV.ES/INDEX.PHP/LEEME HTTP://MUSICA.REDIRIS.ES ISSN: 1575-9563 EDITORES: UNIVERSIDAD DE VALENCIA Y JESÚS TEJADA GIMÉNEZ. VISIBILIDAD DE ESTA REVISTA: EBSCO, CINDOC (CESIC), CITEFACTOR, COPAC, DIALNET, DICE (CSIC), DOAJ, E-REVISTAS (CSIC), EBSCO DOFIDATENBANK, ESTA REVISTA ESTÁ PUBLICADA CON EL APOYO INSTITUCIONAL DE UNIVERSITAT DE VALĖNCIA Y REDIRIS-CONSEJO SUPERIOR DE INVESTIGACIONES CIENTIFICAS Y ES DE ACCESO LIBRE. 
Por este motivo, muchos de ellos explican algunos contenidos muy por encima, dejando muchas dudas y lagunas al respecto. Muchos de los autores sólo exponen sus composiciones virtuosas sin dar ninguna pauta descriptiva al respecto, cuyo único objetivo será la exaltación de la técnica del Violín al servicio del virtuosismo.

Tras el análisis de los métodos propuestos, se ha podido observar que todos los tratados analizados coinciden en que transmitir de una manera clara y concisa los mecanismos tanto físicos como emocionales dentro del arte de la pedagogía del Violín es algo muy complejo, siendo imprescindible tratar de contar con la ayuda de un buen profesor que guíe, explique y aclare todos los contenidos y los aspectos que se encuentran en el manual.

Durante el siglo XIX el estudio del Violín se basaba en la imitación directa del profesor, que a su vez transmitía su enseñanza tratando de clarificar al alumno los contenidos de la técnica del Violín gracias al uso de un determinado método o de una determinada línea metodológica, que posteriormente se transformaría en un método o tratado que serviría de guía al alumno principiante. Por tanto, no es de extrañar que tratados como el de Kreutzer o el de Kayser no se describan los ejercicios propuestos, siendo meramente un compendio de obras compuestas por dichos autores que formarían parte de su programación didáctica, cuyo objetivo era tratar de desarrollar la técnica del Violín de una forma completa.

En conclusión, durante el siglo XIX se produjo un amplio desarrollo de la técnica del Violín gracias fundamentalmente al virtuosismo, este hito marcó la historia de la música e hizo que surgieran ciertas formas musicales de extremada dificultad como los "caprichos", los conciertos o el repertorio de música de cámara. Este fue el principal motivo que hizo que la técnica del instrumento se adaptó a esta nueva manera de tocar con tantas pirotecnias y filigranas interpretativas y a la búsqueda por parte de los maestros de encontrar elementos de apoyo que fueran de ayuda al desarrollo integral de la técnica del Violín. Dicha búsqueda provocó que los maestros del siglo XX y los actuales maestros del siglo XXI den especial importancia a la práctica y al estudio eficiente y de calidad para lograr los máximos resultados en el menor tiempo indagando en la educación postural y en el desarrollo de unos hábitos libres de tensión gracias a la libertad de movimientos y a la concienciación de que el dominio técnico con el Violín está en estrecha relación con el dominio consciente de todos los movimientos realizados en la acción de tocar (Lorenzo y Correa, 2015). 


\section{Referencias}

Alard. D. (1846). École du violon. París : Schott Fréres.

Baillot, P. (1835). L’art du violon, París: Departament Central de la Musique.

Baillot, P., Rode, P., y Kreutzer R. (1974). Méthode de violon, Ginebra: Minkoff.

Brown, C. (2007). Golpes de Arco (1750-1900). Quodlibet, 28, 3-26.

Bruni, A.B. (1804). Méthode pour Ie violon composee sur l'alphabet musical de M. Duhan. París: Departament Central de la Musique.

Dancla, C. (1855). Méthode elementaire et progressif du violon, op. 52, París: Ricordi.

David, F. (1863). Violin school. Nueva York: Oliver Ditson Company.

De Bériot, Ch. (1858). Méthode du violon. París: Schott.

Domínguez, C. O. (2002). Contenidos procedimentales en la pedagogía del violín, su análisis en los métodos Suzuki-Havas-Spiller. Cuadernos Interamericanos de Investigación en Educación Musical, 002-003, 81-105.

Dont, J. (1849). 24 Exercices pour le Violon preparatoires aux Etudes R. Kreutzer y P. Rode. Londres: Augener and Co.

Frega, A. L. (1997). Metodología comparada de la educación musical. Buenos Aires: CEIM del Collegium Musicum.

Garde, A. y Gustems, J. (2014). Ficha de análisis histórico-didáctico para métodos de iniciación al violín. Recuperado de http://diposit.ub.edu/dspace/handle/2445/60685

Garde, A., Gustems, J., y Calderón, C. (2015). Tratados y enseñanza inicial del violin en el siglo XVIII, $60,7-25$.

Geminiani, F, (1751). The Art of Playing on the Violin. Londres: Johnson.

Habeneck, F. A. (1842). Méthode theoriqué et practique. París: Schott.

Hohmann, C. H. (1840). Praktische Violin-schule. Mainz: Schott.

Jorquera, M. C. (2004). Métodos históricos o activos en educación musical. Revista Electrónica de LEEME, 14, 1-55. Recuperado de http://musica.rediris.es/leeme/revista/jorquera04.pdf

Kayser, H. E. (1848). Thirty-six Etudes, op. 20. Elementary and Progressive Studies for the violin. Nueva York: Schirmer.

Kolneder, W. (1998). The Amadeus Book of the Violin: Construction, History, and Music. Cambridge: Amadeus Press.

Lorenzo, O. y Correa, E. (2015). Estudio comparativo de métodos internacionales de enseñanza aprendizaje del violín. Eufonía, 63, 59-66. 
Mazas, J. F. (1843). 75 Études mélodiques et progressives, op. 36 (vol. 1: Études spéciales). Leipzig: Schirmer.

Prieto, C. (1998). Las aventuras de un violonchelo: historia y memorias. México: Fondo de Cultura Económica.

Schradieck, H. (1900). The school of violin-technics, Book I", Milwakee: Schirmer's library of musical classics.

Sevçik, O. (1901). School of violin technique, Op. 1, Part 4, Londres: Bosworrth.

Spohr, L. (1852). Violin School. Boston: Oliver Ditson.

Stowell, R. (Ed.) (1992). The Cambridge Companion to the Violin. Cambridge: Cambridge University Press.

Triviño, L. (2015). Fundamentos expresivos y trascendencia violinística de la escuela parisina de Giovanni D. Viotti, Sevilla: Punto Rojo Libros.

Wohlfart, F. (1874). Sixty Studies for the violin, op. 45, Book I. Nueva York: Schirmer. 